\title{
Pak-Hang Wong:
}

\section{The 'Good Life' in Intercultural Information Ethics: A New Agenda}

\begin{abstract}
:
Current research in Intercultural Information Ethics (IIE) is preoccupied, almost exclusively, by moral and political issues concerning the right and the just (e.g., Hongladarom \& Ess 2007; Ess 2008; Capurro 2008) These issues are undeniably important, and with the continuing development and diffusion of ICTs, we can only be sure more moral and political problems of similar kinds are going to emerge in the future. Yet, as important as those problems are, I want to argue that researchers' preoccupation with the right and the just are undesirable. I shall argue that IIE has thus far overlooked the issues pertaining to the good life (or, individual's well-being). IIE, I claim, should also take into account these issues. Hence, I want to propose a new agenda for IIE, i.e. the good life, in the current paper.
\end{abstract}

\section{Agenda:}

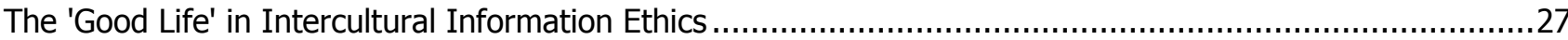

Intercultural Information Ethics and the Minimal Moral Denominator ..........................................22

Putting the Good Life Aside: Pluralism and Subjectivity ...........................................................28

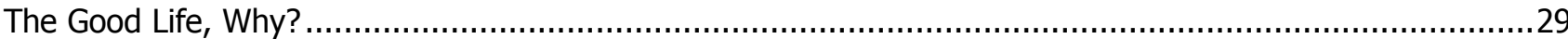

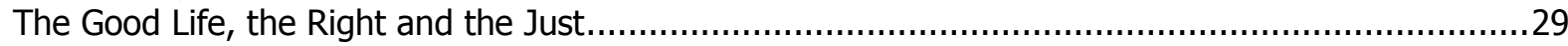

Intercultural Information Ethics and Ameliorative Aspects of ICTs ...........................................30

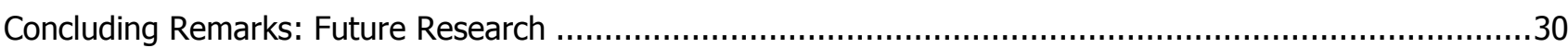

\section{Author:}

Pak-Hang Wong:

- Department of Philosophy, University of Twente, Postbus 217, 7500 AE Enschede, The Netherlands

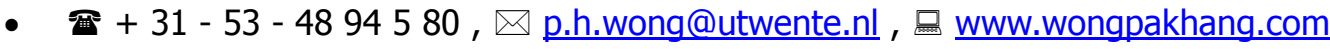

- Relevant publications:

Wong, P.H. (2009): What Should We Share? Understanding the Aim of Intercultural Information Ethics, SIGCAS Computers and Society, 39 (3), 50-58. 
Current research in Intercultural Information Ethics (IIE) is preoccupied, almost exclusively, by moral and political issues concerning the right and the just (e.g., Hongladarom \& Ess 2007; Ess 2008; Capurro 2008). For example, researchers in the field have paid special attentions to privacy (e.g., Ess 2005; Lü 2005; Kitiyadisai 2005; Nakada \& Tamura 2005; Capurro 2005; Hongladarom 2007), intellectual property rights (e.g., Burk 2007), freedom of information (e.g., Rooksby \& Weckert 2007), etc. These problems are undeniably important, and with the continuing development and diffusion of ICTs, we can only be sure more moral and political problems of similar kinds are going to emerge in the future. Yet, as important as those problems are, I want to argue that researchers' preoccupation with the right and the just are undesirable. I shall argue that IIE has thus far overlooked the issues pertaining to the good life (or, individual's well-being). IIE, I claim, should also take into account these issues. Hence, I want to propose a new agenda for IIE, i.e. the good life, in the current paper.

In the first section, I will diagnose the reasons for the negligence of the good life in IIE. Particularly, I will point out that specific understandings of ethical pluralism and subjectivity of the good life may lead researchers in IIE to neglect the issues pertaining to the good life. In the second section, I will demonstrate the importance of the good life in IIE. Firstly, I will argue that the good life is inherently connected to the right and the just, and therefore it cannot be ignored by researchers who are interested in the right and the just. Secondly, I will argue that framing IIE in terms of 'moral problems' and/or 'political problems' have an undesirable distorting effect. The scope of IIE, I will argue, can be broadened to include other important ethical issues by introducing the good life into the field. Finally, I shall end this paper by proposing some directions for future research.

\section{The 'Good Life' in Intercultural Information Ethics}

Charles Taylor has claimed that contemporary moral philosophy "tended to focus on what is right to do rather than on what is good to be, on defining the content of obligation rather than the nature of the good life", and, thus, he lamented that contemporary moral philosophy has become "cramped and truncated." ${ }^{1}$ Similarly, Axel Honneth has recently noted that

"In the last three decades, social criticism has essentially limited itself to evaluating the normative order of societies according to whether they fulfill certain principles of justice. Despite the success of this approach [...], this approach has lost sight of the fact that a society can demonstrate a moral deficit without violating generally valid principles of justice. ${ }^{2}$

Honneth termed the questions left out by social criticism "ethical" questions, which are about what is "desirable beyond all consideration of what is just". ${ }^{3}$ In other words, both Taylor and Honneth have pointed to an existing gap in contemporary moral philosophy and social criticism (or political philosophy). What goes beyond the right for Taylor and the just for Honneth is precisely the issues pertaining to the good life. The issues, which are currently left out, can be succinctly captured by the question: 'How should I live?'4

Whether Taylor's characterisation of contemporary moral philosophy and Honneth's report of the current state of social criticism (or political philosophy) are true in general, I think, they have accurately described the current research in IIE. Researchers in IIE have thus far been shied away from topics beyond what is right and what is just. As a result, I think, the scope of IIE has become unnecessarily limited. In the following section, I will diagnose the main reasons behind the reluctance to incorporate the good life in IIE.

\section{Intercultural Information Ethics and the Minimal Moral Denominator}

Elsewhere, I have attempted to show that the aim of current research in IIE is to establish the minimal moral denominator (MMD), i.e. a set of basic norms and/or normative principles for ethical and political

\footnotetext{
Taylor, Charles: Sources of the Self. 3

Honneth, Axel, Reification. 84

Ibid

Usually, the question: 'How should I live?' is being used in contrasting the right, i.e. morality in the narrow sense, with the good, i.e. morality in the broad sense. (Williams 1985) It is less often to be used in contrasting the the just with the good because the question is individualistically phrased with ' $\mathrm{I}$ '. To overcome the individualistic bias, I think, one may rephrase the question into 'how should a group of individuals, e.g., citizens, live?'
} 
issues which is potentially acceptable by 'all' ethical and cultural traditions. ${ }^{5}$ (Wong 2009) Understanding the aim of IIE to be to establish MMD has a natural tendency to exclude the issues pertaining to the good life because MMD is ill-suited to answer the issues.

I have argued that for MMD to be potentially acceptable by all ethical and cultural traditions, it must be either open or thin, that is - it is either vague and/or ambiguous enough for multiple interpretations, or it is only procedurally justified but not substantively justified. Yet, it is precisely the openness and/or thinness of MMD that make it unsuitable for answering the issues pertaining to the good life. Answering the question: 'How should I live?' normatively requires researchers to offer positive recommendation to individuals. It requires researchers to determine, for example, whether an ICT or ICTs-related activities is good for the individuals and, thus, they should have/use/engage with it.

Yet, MMD, characterised by openness or thinness, is essentially agnostic to positive recommendation. For instance, while MMD's openness allows multiple interpretations of norms and/or normative principles to be valid, it also strips away MMD's ability to determine which interpretation is superior. More importantly, because of the openness of norms and normative principles, MMD cannot non-arbitrarily demonstrate that one positive recommendation is better than another. The same is true of MMD's thinness. Procedural justification is fit to delimit behaviours and social practices, but it suffers from the difficulty to offer positive recommendation. A reasonable procedural justification can tell us what we should not do by ruling out behaviours and social practices with procedures that are accepted by individuals who are minimally rational and by ethical and cultural traditions which satisfy some criteria of reasonableness. In other words, so long as the behaviours and social practices are not ruled out by the procedures, they are permissible. However, the procedures in themselves do not offer any criteria or mechanisms to distinguish the goodness of various behaviours and social practices.

5 To say that the minimal moral denominator (MMD) has to be potentially acceptable by all ethical and cultural traditions is oversimplified and naïve. Instead of all ethical and cultural traditions, MMD is sufficient if it is potentially acceptable by all reasonable ethical and cultural traditions. So construed, however, it implies that a proper and complete formulation of MMD requires a prior account of reasonableness (of ethical and cultural traditions). To avoid further complexities of defining reasonableness, I shall only use the simplistic and naïve formulation of MMD in the current paper.
To sum up: I have argued elsewhere that the aim of current research in IIE is to establish MMD, which is characteristically open and/or thin. The openness and thinness of MMD, in turns, make it difficult to include the good life into IIE because the issues pertaining to the good life are out of reach for MMD.

\section{Putting the Good Life Aside: Pluralism and Subjectivity}

Still, it is worth pondering why researchers in IIE have focused on MMD. There are, I think, two closely related presumptions held by researchers in IIE, each of them has made it difficult for the good life to enter in IIE. Since there are few discussions on the place of the good life in IIE, this section will be largely speculative. ${ }^{6}$ Here, I shall outline the two presumptions, i.e. ethical pluralism and subjectivity of the good life, and explain how specific understandings of them may lead to the omission of the good life in IIE.

As Ess (e.g., 2006, 2007) has argued convincingly, ethical pluralism should be at the core of IIE. Here, ethical pluralism refers to the view that there are different, but equally legitimate ethical and cultural traditions in the world. And, it is the recognition of the truth of ethical pluralism that motivates research in IIE. In recognising the legitimacy of other ethical and cultural traditions, one cannot impose the normative standard of their own tradition to people of other traditions without, at the same time, being guilty of ethical-cultural imperialism. Despite its importance to IIE, specific understanding of ethical pluralism, I suspect, may be responsible for the exclusion of the good life in IIE.

The specific understanding of ethical pluralism in question is best captured by John Rawls's "fact of reasonable pluralism", which stated that we are living in a world characterised by "a pluralism of comprehensive religious, philosophical, and moral doctrines [and, more importantly,] a pluralism of incompatible yet reasonable comprehensive doctrines." Rawls stated that a comprehensive doctrine includes "conceptions of what is of value in human

6 A notable exception is Brey (2007a). Brey has discussed the differences in various value systems and noted that Eastern cultures, e.g., Confucian and/or Buddhist cultures, exhibit different views of the good life. Another exception is, Bynum's (2006) 'Flourishing Ethics', in which the good (or, in Bynum's terms, flourishing) plays a central role. While Flourishing Ethics is not developed explicitly for IIE, it can be extended to IIE. (See, e.g., Ess 2007)

Rawls, John: Political Liberalism. xvi; my emphasis. 
life, and ideals of personal character, as well as ideals of friendship and of familial and associational relationships, and much else that is to inform our conduct, and in the limit to our life as a whole. ${ }^{\prime 8}$ In short, it is people's comprehensive doctrine which informs them how they should live.

In Rawls's view, the goal of political philosophy is to achieve an overlapping consensus. Since reasonable comprehensive doctrines can be incompatible, they have to be set aside. Comprehensive doctrines, thus, are being excluded from political philosophy; and, relatedly, because answers to the question: 'How should I live?' are based on people's comprehensive doctrine; the question, therefore, is not a legitimate consideration in political philosophy, too. Accordingly, one can discern a sharp distinction between the right (and the just) and the good in Rawlsian ethical pluralism. Understanding ethical pluralism in the Rawlsian way, thus, naturally excludes the issues pertaining to the good life because they are not regarded as the proper subjects to political philosophy. While Ess's own view of ethical pluralism strives to overcome the deficiency of Rawlsian ethical pluralism by reintroducing ethical and cultural traditions into IIE, his view resembles Rawlsian ethical pluralism with its primary emphasis on the right (and the just). ${ }^{9}$

A related presumption that leads researchers to exclude the issues pertaining to the good life, I think, is the view that the good life is subjective. This presumption ties closely to the liberal view of person as autonomous and rational being. According to this view, individuals are self-determining and self-responsible for their life project, and they are themselves also the only persons who have authority over their choices. The view also states that individuals are capable of pursuing their life project via means that are most suitable and efficient to them. Together, this view of person asserts the individuals themselves are the only and the best persons with respect to the issues pertaining to their lives. Hence, no inference on their choices of life project is justifiable.

\section{Ibid. 13}

9 Ess, in his recent works (e.g., Ess 2010), has duly noted the importance of the good life in IIE from a virtue ethics approach. While Ess's attempt is admirable, his discussions of IIE remain mostly about the issues on the right and the just. In this way, although the virtue ethics approach has given more attentions to the good, the good still appears to subsume under the right and the just.
In line with the liberal view of person, the issues pertaining to the good life are out of reach from moral and political philosophy. Because of the alleged subjectivity of the good life, it is, therefore, difficult for the researchers in IIE, who hold a broadly liberal view of person, to incorporate the good life into their ethical and political reflections.

Of course, the liberal view of person and the subjectivity of the good life are not unquestioned in moral and political philosophy. ${ }^{10}$ And, indeed, researchers in IIE have explicitly argued for alternatives to the liberal view of person (e.g. Hongladarom, 2007). However, the effort is put mainly to argue for the compatibility of the alternatives to the liberal view of person. In this way, the issues pertaining to the good life are deemed unimportant.

\section{The Good Life, Why?}

In the previous section, I have postulate why issues pertaining to the good life have been excluded in IIE. In this section, I want to offer two claims for including the good life in IIE. My first claim looks at the relationship between the good life, the right and the just, and my second claim focuses on the advantages of incorporating the good life in IIE.

\section{The Good Life, the Right and the Just}

The omission of the good life in current research in IIE, as I have attempted to demonstrated, is a result of the sharp distinction between the right/the just and the good. The separation of the right/the just from the good is, in my view, unwarranted. As Keller (2009) has rightly pointed out, the distinction between the right/the just and the good is merely technical: It does not accord to our pre-theoretical intuition. He has also rightly noted that one's theory of the good will always play a central role in their theory of the right. Consider, for example, Rawls's theory of justice (or, any Rawlsian theory of justice), which is dependent on the notion of primary goods, Rawls's theory of justice is applicable only if a prior list of primary goods is provided. In other words, if we understand 'the good life' as the basic, fundamental components for individuals to live a good life, then the distinction between the right/the just and the good will simply collapse.

10 For an overview, see Wall (2008) and Christman (2009). 
Yet, researchers in IIE may question the aforementioned claim and state that what is at stake is only terminological. Indeed, they can agree entirely with the claim that 'the good life' is important to IIE insofar as it is regarded as the basic, fundamental components for individuals to live a good life, but, at the same time, point out that it adds nothing new to the debate. They may continue to state that the issues have already been taken care of in terms of 'the right' and 'the just', namely if privacy is the basic, fundamental components for individuals to live a good life, then IIE have already discussed the privacy-related problem extensively. Similarly, moral and political problems arise from intellectual property rights, freedom of information, etc. are also widely discussed by researchers in IIE. In other words, researchers in IIE have already taken up the issues, albeit in different terminologies.

I think the complaint is reasonable. It is, indeed, true that privacy, intellectual property rights, freedom of information, etc. are central topics in IIE. Yet, I think, reconceptualising IIE in terms of the good life (in the aforementioned sense) can act as a heuristic device to counter the tendency of reflecting in terms of MMD. Unlike MMD, which aims at delimiting behaviours and social practices, 'the good life' is positive, i.e. it identifies what individuals should have/use/engage with. Hence, it facilitates ethicalpolitical reflections beyond the right and the just.

\section{Intercultural Information Ethics and Ameliorative Aspects of ICTs}

Besides, the current research in IIE too often focuses on the moral and political problems emerge from the development and diffusion of ICTs. The emphasis on 'problems', I think, has created an unfortunate impression that ICTs are merely sources of moral and political disruption. Despite the moral and political challenges brought by ICTs and ICTsrelated activities, we can hardly deny that ICTs are also beneficial to individuals, too. By framing IIE in terms of moral and political problems, researchers in IIE have left out a series of important issues. For example, the Internet, particularly the Social Networking Sites (SNS), has enabled non-face-to-face intimate relationships (e.g. Briggle 2008). Whether this new form of intimate relationship is good for individuals of different ethical and cultural traditions has remained unexplored. Similarly, individuals' (self-) presentation on the Internet has enormous impacts on their life, and the impacts are clearly dependent on the ethical and cultural traditions of the individuals. ${ }^{11}$ The issue of what the better (self-) presentation is in and for different ethical and cultural traditions is indispensible to answer the question: 'How should I live?'

These questions are distinct from moral and political problems in that they are not moral and political problems per se. They have to do with "the best thing to do", whereas in moral and political philosophy the problems are centred on rightness and justice. A failure to do the best thing does not make a person wrong or unjust (at least, not "wrong" in the moral sense). Hence, they are not about permissibility, but about amelioration. In other words, they are about improving one's life by better uses of ICTs and engagements with ICTs-related activities. By incorporating the ameliorative questions into IIE, researchers can go beyond MMD and offer positive recommendation to individuals.

\section{Concluding Remarks: Future Research}

I have argued in this paper that the issues pertaining to the good life have thus far been neglected by researchers in IIE. I have also argued that the negligence is a result of the aim of IIE to be to establish MMD. Finally, I have pointed out that researchers in IIE have no reasons to shy away from issues pertaining to the good life because (i) there is no sharp distinction between the good and the right/the just, and (ii) ameliorative questions of ICTs and ICTs-related activities require inter-/crosscultural investigation, too.

As Brey (2007b) has pointed out, researchers in Information Ethics have only recently begun to look beyond the right and the just. He has also noted that different theoretical framework(s) is required for investigating issues pertaining to the good life. The same is true for investigating the good life in inter-/cross-cultural settings. So far, researchers in IIE have restricted themselves to moral theories, e.g. deontology, consequentialism and virtue-based ethics. These theories are helpful insofar as we regard 'the good life' as the basic, fundamental components for living; but they are unhelpful when we seek to answer the ameliorative questions. ${ }^{12}$ In The point is similar to Weckert's discussion of offense in Weckert (2007).

12 Consequentialism is, perhaps, an exception because it equates the good with the right/the just. 
order to answer the ameliorative questions, we need to know what is good for 'us'. In other words, we need a theory of the good life (or well-being), which tells us what we should strive for.

In the West, philosophers have elaborated three major theories of the good life, i.e. hedonism, desire theories and objective-list theories. ${ }^{13}$ These theories allow researchers in Information Ethics to evaluate the impacts of ICTs and ICTs-related activities on individual's well-being; and, also allow them to offer positive recommendation based on the theories of the good life they maintain. Unfortunately, there are relatively few contemporary philosophical studies on the non-Western theories of the good life. Hence, to embark on the new agenda of IIE, the first step is to systematically investigate the non-Western theories of the good life. Once the non-Western theories of the good life are elaborated, researchers in IIE can begin to compare and contrast how different ethical and cultural traditions perceive the impacts of ICTs and ICTs-related activities on the good life. Researchers in IIE, then, will be better equipped to offer positive recommendation with respect to the good life in inter-/cross-cultural settings.

The future IIE, therefore, should consist of two parallel strands. The first strand, which has already existed in IIE, focuses on the moral and political problems emerging from the development and diffusion of ICTs. The second strand, then, aims at the ameliorative aspects of ICTs and ICTs-related activities. Among other things, IIE of the good life investigates theories of the good life of different ethical and cultural traditions; and it attempts to improve individual's well-being in inter-/crosscultural settings. ${ }^{14}$

\section{References}

Brey, P. (2007a) Is Information Ethics CultureRelative? International Journal of Technology and Human Interaction, 3(3), 12-2.

Brey, P. (2007b) Theorizing the Cultural Quality of New Media. Techne, 11, 2-18.

13 It is beyond my scope to provide an in-depth analysis of different philosophical theories of the good life/well-being in this paper. For an overview of these theories, see Crisp (2006), Hurka (2006) \& Schroeder (2008).

14 Early version of this paper was presented at ReGIS $\times$ STI-IE Joint-Workshop on Intercultural Information Ethics in Asia (University of Tsukuba (Japan), 2009). I would like to thank my audiences for helpful remarks and suggestions. Particularly, I would like to thank Prof. Rafael Capurro and Prof. Makoto Nakada for their valuable comments.
Briggle, A. (2008) Real Friends: How the Internet Can Foster Friendship. Ethics and Information Technology, 10. 71-79.

Burk, D. (2007) Privacy and Property in the Global Datasphere. In C. Ess and S. Hongladarom (eds.), Information Technology Ethics: Cultural Perspectives (pp. 94-107), Hershey, PA: Idea Group Reference.

Bynum, T. (2006) Flourishing Ethics. Ethics and Information Technology, 8, 157-173.

Capurro, R. (2008) Intercultural Information Ethics. In Einar Himma and Herman T. Tavani (eds.), Handbook of Information and Computer Ethics (pp. 639-665). Hoboken, New Jersey: John Wiley \& Sons.

Capurro, R. (2005) Privacy. An Intercultural Perspective. Ethics and Information Technology, 7, 3747.

Christman, J. (2009). Autonomy in Moral and Political Philosophy. The Stanford Encyclopedia of Philosophy (Fall 2009 Edition). URL = <http://plato.stanford.edu/archives/fall2009/ent ries/autonomy-moral/>

Crisp, R. (2008). Well-Being. The Stanford Encyclopedia of Philosophy (Winter 2008 Edition). URL $=$ <http://plato.stanford.edu/archives/win2008/en tries/well-being/>

Ess, C. (2010) Brave New Worlds? The Once and Future Information Ethics. International Review of Information Ethics, 12, 36-44.

Ess, C. (2008) Culture and Global Networks: Hope for a Global Ethics? In J. van den Hoven and J. Weckert (eds.), Information Technology and Moral Philosophy (pp. 195-225), Cambridge: Cambridge University Press.

Ess, C. (2007) Cybernetic Pluralism in an Emerging Global Information and Computing Ethics. International Review of Information Ethics, 7, 1-31.

Ess, C. (2006) Ethical Pluralism and Global Information Ethics. Ethics and Information Technology, 8, 215-226.

Ess, C. (2005)"Lost in Translation"?: Intercultural Dialogues on Privacy and Information Ethics (Introduction to Special Issue on Privacy and Data Privacy Protection in Asia). Ethics and Information Technology, 7, 1-6.

Ess, C. and Hongladarom, S. (2007). Information Technology Ethics: Cultural Perspectives. Hershey, PA: Idea Group Reference.

Hongladarom, S. (2007) Analysis and Justification of Privacy from a Buddhist Perspective. In C. Ess and $S$. Hongladarom (eds.), Information Tech- 
nology Ethics: Cultural Perspectives (pp. 108122), Hershey, PA: Idea Group Reference.

Honneth, A. (2008) Reification : A New look at An Od Idea. Oxford; New York: Oxford University Press.

Hurka, Thomas. (2006). Value-Theory. In D. Copp (ed.), The Oxford Handbook of Ethical Theory (pp.357-79). New York: Oxford University Press.

Keller, S. (2009) Welfarism. Philosophy Compass 4(1). 82-95.

Kitiyadisai, K. (2005) Privacy Rights and Protection: Foreign Values in Modern Thai Context. Ethics and Information Technology, 7, 17-26.

Lü, Yao-Huai. (2005) Privacy and Data Privacy Issues in Contemporary China. Ethics and Information Technology, 7, 7-15.

Nakada, M. \& Tamura, T. (2005) Japanese Conceptions of Privacy: An Intercultural Perspective. Ethics and Information Technology, 7, 27-36.

Rooksby, E. \& Weckert, J. (2007) Information Technology and Social Justice, Hershey, PA: Idea Group Reference.

Schroeder, Mark. (2008). Value Theory. The Stanford Encyclopedia of Philosophy (Fall 2008 Edition). URL

$<$ http://plato.stanford.edu/archives/fall2008/ent ries/value-theory/>
Taylor, C. (1989). Sources of the self: the making of modern identity. Cambridge: Cambridge University Press.

Wall, S. (2008). Perfectionism in Moral and Political Philosophy. The Stanford Encyclopedia of Philosophy (Fall 2008 Edition). URL = <http://plato.stanford.edu/archives/fall2008/ent ries/perfectionism-moral/>

Weckert, J. (2007) Giving and Taking Offence in a Global context. International Journal of Technology and Human Interaction, 3, 25-35.

Williams, B. (1985) Ethics and the Limits of Philosophy, London: Fontana.

Wong, P.H. (2009) What Should We Share? Understanding the Aim of Intercultural Information Ethics. SIGCAS Computers and Society, 39 (3), 50-58. 